\title{
The influence of haptic size information upon visual judgments of absolute distance
}

MAX COLTHEART'
MONASH UNIVERSITY

When $O$ views a blank triangle of light under completely reduced conditions, he is able to use information about the size of this visual stimulus conveyed via the haptic modality when he is attempting to judge the absolute distance of the visual stimulus. However, distance is consistently underestimated in this situation. When haptically-indicated size is held constant, judged distance varies inversely with retinal subtense, even though the different retinal subtenses are viewed by different $O s$. A variant of the size-distance invariance hypothesis also appears to hold in these circumstances.

Previous experiments (Coltheart, 1968, and unpublished) indicated that information about the size of a visual stimulus, whether this is contributed in the form of assumed size or of familiar size, may be utilized by $O$ s when they make judgments of the absolute distance of the stimulus under completely reduced conditions. The aim of the present experiment was to establish whether another form of size information, namely haptic size information, is also capable of influencing visual judgments of absolute distance.

\section{METHOD}

The apparatus is described fully elsewhere (Coltheart, 1968) and will only be described briefly here. The Os (undergraduates at the University of Sydney) were led blindfolded into a light-tight dark room measuring $17 \mathrm{ft} \times 32 \mathrm{ft}$, and seated in one corner. The blindfold was removed and $\mathrm{O}$ sighted across towards the diagonally opposite corner of the room, using his preferred eye and looking through a monocular viewing tube in a Masonite screen. His line of sight passed through a 5 -in. $x$ 3 -in. elliptical aperture in a reduction screen, 36 in. from O's eye.

Fourteen feet from $O$ and at his eye level was the visual stimulus, an illuminated equilateral triangle produced by cutting out a triangle from the opaque material masking the milk-glass screen of a light-box. While $O$ was viewing this visual stimulus, E handed him the haptic stimulus, an equilateral triangle made of Masonite, and instructed $\mathrm{O}$ to hold it in both hands. It was never visible to $O$. The following instructions were then given:

"The triangle you are holding is exactly the same size and shape as the triangle you are looking at. I want you to tell me how far the triangle you are looking at is from you; how far is it in feet and inches to that triangle? Remember that it is the same size and shape as the triangle you are holding."

Visual stimuli of two sizes and haptic stimuli of two sizes were used so that four groups of Os were needed. The various experimental conditions are shown in Table 1.

The method of planned contrasts (Hays, 1963; Rodger, 1967) was used in designing the experiment. According to this method, no more than four hypotheses concerning population

Table 1

Experimental Conditions for the Four Groups

\begin{tabular}{ccc}
\hline Group & $\begin{array}{c}\text { Length of Side } \\
\text { of Haptic Stimulus }\end{array}$ & $\begin{array}{c}\text { Length of Side } \\
\text { Of Visual Stimulus }\end{array}$ \\
\hline 1 & $4 \mathrm{in.}$ & $4 \mathrm{in.}$ \\
2 & $8 \mathrm{in.}$ & $4 \mathrm{in.}$ \\
3 & $4 \mathrm{in.}$ & $8 \mathrm{in.}$ \\
4 & $8 \mathrm{in.}$ & $8 \mathrm{in.}$ \\
\hline
\end{tabular}

Table 2

Hypotheses to be Tested by the Method of Planned Comparisons

Hypotheses Effect Tested

$\mathrm{H} 1: 1 / 2(\mu 1+\mu 2)-1 / 2(\mu 3+\mu 4)=0$ Main effect of size of visual stimulus $\mathrm{H} 2: 1 / 2(\mu \mathrm{l}+\mu 3)-1 / 2(\mu 2+\mu 4)=0$ Main effect of size of haptic stimulus H3: $\mu 1-\mu 4=0$

$\mathrm{H} 4: \frac{1}{2}(\mu 1+\mu 4)-14=0$ Invariance hypothesis Veridicality of the haptic cue

means can be tested if the experiment involves four groups, and of these four hypotheses, at most three may be contrasts across the means. The hypotheses which were selected for testing, before gathering any data, are shown in Table 2.

$\mathrm{H} 1$ and $\mathrm{H} 2$ are the usual tests of the main effects in a 2 by 2 factorial design. $\mathrm{H} 3$ was a test of the (haptic) size (visual) distance invariance hypothesis. Since the ratio of hapticstimulus size to retinal angle of the visual stimulus was the same for Groups 1 and 4 , this form of invariance hypothesis would predict no difference between the distance judgments of the two groups. H4 (which does not involve a contrast) tested the veridicality of the haptic cue; Groups $I$ and 4 were the only groups which received veridical haptic information about the size of the visual stimulus, and if this information were used to make veridical judgments, the population mean distance judgment would for both groups be $14 \mathrm{ft}$, which is the actual distance; therefore, a test of the hypothesis that the average of the two means is $14 \mathrm{ft}$ is an overall test of the veridicality of the haptic cue.

Different Os were used in each of the four groups, and the minimum number per group required to impart adequate sensitivity to tests of the hypotheses was calculated by the methods described by Guenther (1965) and Rodger (1967). In a previous experiment using disc stimuli with an otherwise identical experimental arrangement, the MSW of distance estimates was a little less than 30 , and in another experiment with rectangular stimuli MSW for distance estimates was 37.4. It was therefore considered reasonable to use a value of 36 as an estimation of the population variance of distance estimates of triangular stimuli.

Given this, calculations indicate that a minimum sample size of 20 is sufficient to yield a probability of .95 (Type II error rate of .05 ) of detecting an effect of $5 \mathrm{ft}$ in the population. That is, if the combination of population means defined in $\mathrm{Hl}$ or $\mathrm{H} 2$ did not have a value of 0 , as the hypothesis asserts, but of $5 \mathrm{ft}$, the probability of falsely accepting $\mathrm{H}$ would be .05 .

In fact, 88 Os were run, 22 per group, thus increasing the sensitivity of the experiment a little more.

\section{RESULTS}

Means and standard deviations of distance estimates for the four groups are given in Table 3. The MSW was 36.26.

The results of testing the four hypotheses are shown in Table 4. The second column of this table shows the value which the contrast would have to have in the sample to be significant at the .05 level and the third column shows the obtained value.

The average of the means of Groups 1 and 4 was significantly less than $14 \mathrm{ft}$, i.e., $\mathrm{H} 4$ was rejected $(t=4.97$. df $=43, p<.001$ ). 
Table 3

Means and SDs of Distance Estimated for each Group

\begin{tabular}{lcc}
\hline Group & Mean Estimated Distance $(\mathrm{ft})$ & $\mathrm{SD}(\mathrm{ft})$ \\
\hline 1 & 9.16 & 6.42 \\
2 & 12.36 & 5.78 \\
3 & 5.87 & 3.85 \\
4 & 9.82 & 7.07 \\
\hline
\end{tabular}

\section{DISCUSSION}

The rejection of $\mathrm{H} 2$ indicated that when the retinal subtense of a visual stimulus is held constant, the groups having the larger haptic comparison-object yield larger distance estimates. This is what would be expected if Os were able to take haptic size information into account when judging absolute distance; if retinal size is fixed, the objectively larger stimulus must be farther away.

The retention of $\mathrm{H} 3$ enables one to entertain a form of the size-distance invariance hypothesis according to which the two stimuli having the same ratio of visual angle to (hapticallyinformed) size are judged to be at the same distance.

Although Os did take haptic size information into account when judging absolute distance, they were not able to make veridical judgments of distance; the rejection of $\mathrm{H} 4$ indicates that absolute distance was significantly underestimated when veridical haptic information was supplied.

Table 4

Tests of Contrasts

\begin{tabular}{cccl}
\hline Hypothesis & Critiçal Value & Obtained Value & Decision \\
\hline $\mathrm{H} 1$ & $2.56 \mathrm{ft}$ & $4.07 \mathrm{ft}$ & Reject H1 \\
$\mathrm{H} 2$ & $2.56 \mathrm{ft}$ & $2.92 \mathrm{ft}$ & Reject H2 \\
$\mathrm{H} 3$ & $3.62 \mathrm{ft}$ & $0.66 \mathrm{ft}$ & Retain H3 \\
\hline
\end{tabular}

\section{CONCLUSIONS}

When retinal size is constant, judged distance increases as the size indicated by a haptic comparison-object increases. Therefore, Os are able to take haptically-imparted size information into account when making judgments of distance. However, they are not able to judge distance veridically under these conditions; the true distance of the object being viewed was underestimated.

When haptically-indicated size is held constant, judged distance is decreased when retinal subtense is increased. This is similar to what occurs when there is no size information at all, since in the latter situation judged size does not vary with retinal subtense, whereas judged distance is inversely related to retinal subtense.

When haptically-indicated size and retinal subtense were both doubled in one condition relative to another, judged distance remained constant, indicating that a variant of the size-distance invariance hypothesis is tenable under these conditions.

\section{REFERENCES}

COLTHEART, M. Size information as a factor in visual judgments of absolute distance under reduced conditions. Unpublished $\mathrm{Ph}$. D. Thesis, University of Sydney, 1968.

GUENTHER, W. C. Concepts of statistical inference. New York: McGraw-Hill, 1965.

HAYS, W. L. Statistics for psychologists. New York: Holt, Rinehart, \& Winston, 1963 .

RODGER, R. S. Type II errors and their decision basis. British Journal of Mathematical and Statistical Psychology, 1967, 20, 187-204.

NOTE

1. Address: Department of Psychology, Monash University, Clayton, Victoria 3168 , Australia.

(Accepted for publication September 23, 1968.) 